\title{
RELAÇÕES INTERPESSOAIS ENTRE PROFISSIONAIS DE ENFERMAGEM E FAMILIARES EM UNIDADE DE TRATAMENTO INTENSIVO: ESTUDO BIBLIOGRÁFICO
}

Lissandra Maicá Ruedell ${ }^{1}$, Carmem Lúcia Colomé Beck², Rosângela Marion da Silva ${ }^{3}$, Rosa Ladi Lisboa ${ }^{4}$, Andréa Prochnow ${ }^{4}$, Francine Cassol Prestes ${ }^{4}$

RESUMO: Pesquisa bibliográfica cujo objetivo foi buscar referenciais teóricos que subsidiassem a compreensão dos fatores que influenciam nas relações entre profissionais de enfermagem e familiares de pacientes internados em Unidades de Terapia Intensiva, possibilitando o exercício crítico-reflexivo. Foram selecionados 18 artigos na base Literatura Latino-Americana e do Caribe em Ciências da Saúde. Realizou-se leitura dos textos na íntegra e a construção de categorias temáticas. Os fatores que interferem no relacionamento dos profissionais de enfermagem com a família são: falta de tempo para assistir a família; medo do envolvimento emocional; comportamento impessoal do enfermeiro com o familiar; família percebida como intrusa; e linguagem inadequada utilizada pela enfermagem. Quanto aos fatores que influenciam a família a interagir com profissionais de enfermagem, foi identificado: tensão, insegurança e medo do familiar frente à situação; falta de confiança da família na equipe; ambiente físico desfavorável e rejeição ou negação das informações recebidas.

PALAVRAS-CHAVE: Relações profissional-família; Unidades de terapia intensiva; Cuidados de enfermagem; Prática profissional.

\section{INTERPERSONAL RELATIONSHIP BETWEEN NURSING PROFESSIONALS AND FAMILIES IN THE INTENSIVE CARE UNIT: A BIBLIOGRAPHICAL STUDY}

\begin{abstract}
Bibliographical research in which the objective was to search for theoretical references that subsidize the comprehension of the factors that influence the relationship between nursing professionals and the families of patients hospitalized in Intensive Care Units, making possible the critic-reflexive exercise. 18 articles were selected in the LILACS database (Latin American and Caribbean Literature on Health Science). The full reading of the texts was performed, as well as the construction of thematic categories. The factors that interfere in the relationship of the nursing professionals with the families were: lack of time to assist the families; fear of emotional involvement; impersonal behavior of the nurse with the family; family perceived as an intruder and inadequate language used by the nurses. As for the factors that influence the families to interact with the nursing professionals, tension, insecurity and fear of the families due to the situation were detected, lack of confidence of the family on the staff; unfavorable physical environment and the rejection or denial of the information received.
\end{abstract}

KEYWORDS: Professional-family relations; Intensive care units; Nursing care; Professional practice.

\section{RELACIONES INTERPERSONALES ENTRE PROFESIONALES DE ENFERMERÍAY FAMILIARES EN UNIDAD DE TRATAMIENTO INTENSIVO: ESTUDIO BIBLIOGRÁFICO}

RESUMEN: Investigación bibliográfica cuyo objetivo fue buscar referenciales teóricos que subsidiasen la comprensión de los factores que influencian en las relaciones entre profesionales de enfermería y familiares de pacientes internados en Unidades de Terapia Intensiva, posibilitando el ejercicio crítico-reflexivo. Fueron seleccionados 18 artículos en la base Literatura Latino Americana y del Caribe en Ciencias de la Salud). Se realizó lectura de los textos en la íntegra y construcción de categorías temáticas. Los factores que interfieren en el relacionamiento de los profesionales de enfermería con la familia son falta de tiempo para asistir la familia; miedo de envolvimiento emocional; comportamiento impersonal del enfermero con el familiar; familia percibida como intrusa y lenguaje inadecuada utilizada por la enfermería. Cuanto a los factores que influencian la familia a interactuar con profesionales de enfermería, fue identificadora tensión, inseguridad y miedo del familiar delante a la situación; falta de confianza de la familia en el equipo; ambiente físico desfavorable y rechazo o negación de las informaciones recibidas.

PALABRAS CLAVE: Relaciones profesional-familia; Unidades de terapia intensiva; Atención de enfermería; Práctica profesional.

${ }^{1}$ Enfermeira da Prefeitura Municipal de Gravataí, Rio Grande do Sul.

${ }^{2}$ Enfermeira. Doutora em Enfermagem. Professor Associado I do Departamento de Enfermagem da Universidade Federal de Santa Maria-UFSM. Membro do Grupo de Pesquisa Trabalho, Saúde, Educação e Enfermagem da UFSM.

${ }^{3}$ Enfermeira. Mestre em Enfermagem pela UFSM. Membro do Grupo de Pesquisa Trabalho, Educação, Saúde e Enfermagem da UFSM. ${ }^{4}$ Acadêmica de Enfermagem da UFSM. Membro do Grupo de Pesquisa Trabalho, Educação, Saúde e Enfermagem da UFSM.

Autor correspondente:

Carmem Lúcia Colomé Beck

Universidade Federal de Santa Maria

Rua Coronel Estácio Mariense de Lemos, 154 - 97020-150 - Santa Maria-RS, Brasil

Recebido: 27/03/09

E-mail: carmembeck@smail.ufsm.br

Aprovado: 23/09/09 


\section{INTRODUZINDO A TEMÁTICA}

A Unidade de Tratamento Intensivo (UTI) tem como objetivo principal atender pacientes em estado crítico, na tentativa de possibilitar maiores probabilidades de recuperação, centralizando aparato tecnológico e assistência de saúde especializada. Assim sendo, essas características a transformam em um ambiente estressor para os pacientes, seus familiares e profissionais da saúde que atuam nessa unidade.

O cenário de trabalho na UTI é caracterizado por uma dinâmica diversa dos demais cenários hospitalares; em geral apresenta horários diferenciados para visitas, e o cuidado é marcado pelo uso intensivo de tecnologias e aparelhos de última geração que dão suporte à manutenção da vida, como ventiladores mecânicos, monitores cardíacos, cateteres, dentre outros. Desse modo, os profissionais precisam estar preparados para realizar o cuidado com competência e eficiência, não se deixando dominar pela rotina e pela banalização da assistência, o que poderá tornar suas ações mecânicas e impessoais.

No momento da hospitalização de um paciente na UTI, em especial por ser um ambiente com características peculiares associadas à gravidade do quadro de saúde e à possibilidade da morte do paciente, a família pode experimentar sentimentos como o medo e a insegurança ${ }^{(1)}$.

No que se refere ao cuidado da família pelos profissionais da enfermagem, é essencial percebê-la como uma unidade, assim como observar seus integrantes a partir de seus gestos, falas e comportamentos. Desta forma, torna-se fundamental acompanhar a família do paciente na UTI, no sentido de potencializar o trabalho da Enfermagem, questionando-a sobre dúvidas, observando-lhe as reações e comportamentos, procurando entender suas emoções ${ }^{(2)}$. É necessário envolver a família como parte integrante da prática do cuidado no dia-a-dia, considerando angústias, dúvidas e expectativas, a fim de desenvolver um cuidado congruente com qualidade ${ }^{(3)}$.

Tal envolvimento também pode ser efetivado pelos profissionais de enfermagem no horário da visita, momento em que é favorecida à equipe de profissionais da saúde a percepção e vivência da hospitalização pela família do paciente. Nesse contexto, é esperado que esses profissionais construam vínculos com os familiares, o que pode favorecer sua sensibilização com o sofrimento ${ }^{(4)}$.
É importante que as orientações à família sejam realizadas desde o momento da internação, enquanto elemento essencial para o cuidado e, também, como uma possibilidade de estabelecer relações interpessoais entre a equipe de enfermagem e o grupo familiar. Isso pode favorecer uma comunicação mais efetiva e o compartilhar de significados emergentes diante da situação estressante de ter um familiar na UTI ${ }^{(1)}$.

Os profissionais de enfermagem, nessa perspectiva, têm papel fundamental na adaptação e recuperação do paciente, bem como na assistência à família. Para promover esse vínculo e, consequentemente, um bom relacionamento interpessoal entre o paciente, o profissional e a família, é necessário estabelecer relações harmoniosas, fundamental para o processo de cuidado e cura do paciente. Para tanto, o enfermeiro deve ser um dos profissionais da área da enfermagem capacitado a reconhecer a interação enfermeiro-paciente-família e desenvolver atitudes que demonstrem sensibilidade e empatia, contribuindo para a assistência humanizada ${ }^{(5)}$.

No contexto da hospitalização, é mister que os profissionais de enfermagem entendam os significados e as diferentes formas de ver a saúde e a doença e que, em seu agir, levem em consideração os significados culturais de cada pessoa, e sejam coerentes com as necessidades individuais e familiares ${ }^{(6)}$. Nesse sentido, o entendimento sobre saúde, doença e cuidados, tanto por parte da família quanto dos profissionais de enfermagem, pode divergir, pois são aspectos fortemente influenciados pelas perspectivas culturais de cada pessoa ou grupo.

A partir dessas considerações, esta pesquisa teve como objetivo buscar referenciais teóricos que subsidiassem a compreensão dos fatores que influenciam nas relações entre os profissionais de enfermagem e os familiares de pacientes internados em Unidades de Terapia Intensiva, possibilitando o exercício crítico-reflexivo sobre o tema.

\section{PERCURSO METODOLÓGICO}

Este artigo apresenta uma pesquisa bibliográfica, a qual é utilizada quando se quer explicar um problema ou buscar lacunas do conhecimento, a partir de referências teóricas publicadas em documentos, buscando conhecer ou analisar as contribuições científicas existentes sobre determinado assunto ou tema $^{(7)}$.

O levantamento dos artigos foi realizado no 
segundo semestre de 2007 e retomado e atualizado no primeiro semestre de 2009, por meio da consulta online na base de dados Literatura Latino-Americana e do Caribe em Ciências da Saúde (LILACS).

Foram utilizados como descritores ou palavraschave as expressões isoladas ou em associação: "relações interpessoais", "unidade de terapia intensiva”, "cuidados de enfermagem" e "prática profissional".

Os critérios de inclusão dos textos no estudo foram: ser artigo científico, ter sido desenvolvido em UTI de Adulto, estar disponível online na íntegra e remeter ao objetivo do estudo, com foco na equipe de enfermagem.

A partir dos descritores ou palavras-chave, em uma primeira busca, foram encontradas 315 produções. Dessas, 18 foram selecionadas conforme os critérios de inclusão, a partir da leitura do título e do resumo, as quais contemplavam o objetivo do estudo. Após, realizou-se a leitura dos textos na íntegra, o agrupamento das informações, considerando-se as semelhanças e diferenças, o que possibilitou a construção de duas categorias temáticas.

\section{RESULTADOS}

Ao identificar os autores dos artigos utilizados nesta pesquisa, pode-se dizer, que são majoritariamente docentes de Enfermagem, contando ainda com a participação de enfermeiros assistenciais, alunos de graduação e de um docente de psicologia.

Os dados emergidos da análise qualitativa evidenciaram duas categorias temáticas, a saber: fatores que interferem no relacionamento interpessoal entre profissionais de enfermagem e a família e fatores que influenciam a família ao interagir com os profissionais de enfermagem na UTI.

No que se refere à primeira categoria - fatores que interferem no relacionamento interpessoal entre profissionais de enfermagem e a família - emergiram cinco subcategorias: falta de tempo dos profissionais de enfermagem para assistir a família; medo do envolvimento emocional com a família; comportamento impessoal do enfermeiro no trato com o familiar; a família percebida como intrusa na unidade de internação e a linguagem inadequada utilizada pelos profissionais de enfermagem na abordagem à família.

A primeira subcategoria, falta de tempo dos profissionais de enfermagem para assistir a família, revela a influência do ritmo intenso de trabalho da equipe de enfermagem nas instituições hospitalares.
Percebe-se que os profissionais de enfermagem podem ter dificuldades para prestar um atendimento integral, tendo em vista o número elevado de pacientes, bem como a carência de profissionais para a realização dos cuidados necessários. Essa situação torna-se complexa, considerando-se que a assistência ao paciente não deve ser o único foco do cuidado. Logo, na assistência, o profissional precisa buscar a perspectiva da integralidade, incluindo a família.

Quanto à segunda subcategoria, medo do envolvimento emocional do profissional de enfermagem com a família, foi evidenciado, na análise dos materiais encontrados, enfrentamentos dos profissionais no que tange à interação afetiva com o paciente. Esses enfrentamentos podem se manifestar por meio de sentimentos como o medo, tanto do processo de hospitalização quanto da morte do paciente. No entanto, humanizar o atendimento na situação de dor, sofrimento e morte é revitalizar a relação empática do processo terapêutico, em que a reciprocidade do contato permite o entendimento do sofrimento do outro, bem como a reflexão sistemática e contínua sobre o processo de viver-morrer ${ }^{(8)}$.

A Enfermagem, quando não se encontra preparada para enfrentar o processo da morte e do morrer, pode manifestar afastamento do paciente e de sua família. De outra forma, acredita-se que esse momento possa ser permeado pela interação, ajuda mútua e construção de laços que auxiliarão na vivência de todos os envolvidos nesse processo.

Ressalta-se que a dificuldade de relacionamento causada pelo envolvimento emocional excessivo, ou não-envolvimento, pode ser prejudicial na relação profissional de enfermagem-paciente-família. Sendo assim, há necessidade de promover um ajuste que mantenha o equilíbrio situacional e comportamental nessa relação ${ }^{(5)}$.

Como terceira subcategoria foi encontrado o comportamento impessoal do enfermeiro no trato com a família. O ambiente de uma UTI, para a família, é muitas vezes impessoal e complexo, o que pode favorecer a manifestação de atitudes defensivas, dificultando a formação de elos e de uma comunicação efetiva entre eles e os profissionais de enfermagem.

Nesse aspecto, fica evidente a necessidade de uma comunicação adequada entre a Enfermagem e a família, ampliando-a para além das informações a respeito de normas e rotinas, ou seja, é necessário considerar as reais necessidades psicossociais do paciente e da sua família. Todavia, a repetição das 
informações por parte dos trabalhadores à família, relativas às normas e rotinas, podem indicar um mecanismo de defesa para fazer frente à ansiedade apresentada na hospitalização.

A família percebida como intrusa na unidade de internação foi a quarta subcategoria, sendo destacado, neste aspecto, a influência do ambiente de trabalho nem sempre adequado. Isso porque, em geral, estas unidades têm grande circulação de profissionais da área da saúde, o que inclui os que realizam exames laboratoriais de rotina ou de urgência e, em algumas instituições de saúde, alunos de cursos de graduação e pós-graduação em aulas práticas ou estágios curriculares.

Ainda neste contexto, o sofrimento da família também pode ser identificado quando manifesta curiosidade, necessidade de conhecer e questionar a realização de alguns procedimentos, a evolução clínica dos pacientes, bem como o prognóstico, o que pode desencadear alterações de humor como irritabilidade e desconforto no profissional de enfermagem.

Em muitas situações em que os profissionais estabelecem relações tênues com as famílias, podese identificar a presença de funcionários antigos, efetivos e de instituições públicas, que não dependem da reputação para manter seus empregos ${ }^{(7)}$.

Na quinta subcategoria, linguagem inadequada utilizada pelos trabalhadores da enfermagem na abordagem dos membros da família, foram encontrados estudos que referem que os profissionais utilizam termos técnicos ao se comunicarem com as famílias, sem adaptá-los à linguagem do cotidiano daquele familiar. Isso pode implicar em interpretações errôneas, constituindo-se barreiras na comunicação, as quais são potencialmente geradoras de tensão.

Quanto à segunda categoria, fatores que influenciam a família ao interagir com os profissionais de enfermagem na UTI, foram encontradas quatro subcategorias: tensão, insegurança e medo do familiar frente à situação; falta de confiança da família na equipe de enfermagem; ambiente físico desfavorável; e rejeição ou negação das informações recebidas.

$\mathrm{Na}$ primeira subcategoria, tensão, insegurança e medo do familiar frente à situação, foram identificados estudos que apontam que a família tem medo da morte do familiar internado na UTI, sentindo-se sobrecarregada diante do enfrentamento trazido pela doença e internação hospitalar. Ela também sofre ao experienciar algumas complicações no estado de saúde do paciente; angustia-se diante da demora do diagnóstico; enfrenta, em muitas situações, dificuldades financeiras; tem resistência em aceitar que uma pessoa estranha cuide do seu ente querido e pode apresentar dúvidas com relação ao prognóstico. O familiar do paciente crítico, ao ter consciência da situação concreta e da possibilidade de morte do seu enfermo que está na UTI, pode expressar o vazio existencial por meio de sentimentos como tristeza, frustração, pessimismo, desorientação, angústia e falta de sentido para viver ${ }^{(9)}$.

A falta de confiança da família na equipe de enfermagem foi a segunda subcategoria encontrada. Na maioria das vezes, o fato de deixar um paciente em estado crítico, em um ambiente como a UTI, e tendo consciência do risco de morte, pode representar sofrimento para a família. É pertinente, nesse contexto, que o enfermeiro seja capaz de detectar as necessidades psicobiológicas, psicossociais e psicoespirituais e auxiliar a família e o paciente no atendimento das necessidades que estão afetadas, de acordo com a prioridade, porque ele é um ser dependente dos cuidados da enfermagem ${ }^{(10)}$.

Nesse contexto, todo e qualquer contato com o paciente ou familiar que precisa de ajuda deveria ser terapêutico, sendo fundamental que o enfermeiro busque compreender o comportamento do outro e a percepção dele sobre o que o rodeia. Desse modo, a comunicação efetiva pode possibilitar, ao familiar, enfrentar momentos de incerteza e ansiedade com maior segurança, pautados na confiabilidade, empatia e aceitação ${ }^{(11)}$.

$\mathrm{Na}$ terceira subcategoria, ambiente físico desfavorável, destaca-se a estrutura imprópria de muitas unidades de terapia intensiva, em especial as salas de espera, o que pode dificultar o processo de interação entre os familiares e a equipe de enfermagem dessa unidade. A ausência de um local apropriado é considerada um fator negativo nas relações interpessoais, que pode dificultar o encontro e o diálogo entre trabalhadores e familiares de pacientes internados.

Na quarta subcategoria, rejeição ou negação das informações recebidas por parte dos familiares, a análise dos estudos apontou que há mecanismos de defesa dos familiares no que se refere ao conhecimento do estado de saúde do paciente. Esses são manifestados por atitudes de negação e rejeição diante das informações recebidas pela equipe de enfermagem. As famílias também sentem dificuldade em aceitar as normas estabelecidas pela instituição, como os horários de visitas, a impossibilidade de entrar na UTI a qualquer hora, entre outros. Dessa forma, 
manifestam indignação com os profissionais de enfermagem por serem as pessoas com as quais estabelecem maior interação.

\section{ALGUMAS CONSIDERAÇÕES}

Ao final desta análise, destaca-se a abrangência do papel da equipe de enfermagem que atua em UTI, uma vez que esta apresenta características peculiares, as quais apontam para o aumento das probabilidades de sofrimento psíquico nos profissionais de saúde/ enfermagem, tendo em vista a complexidade das ações realizadas e o estresse que pode ser gerado, tanto no paciente e nos profissionais de saúde, quanto nos próprios familiares.

Na análise dos artigos selecionados, evidenciouse a escassez de abordagens em relação aos aspectos que influenciam a família na interação com os profissionais de enfermagem. Isto pode ocorrer devido à pouca valorização da família neste contexto de práticas de saúde, apontando também para uma necessidade de realização de estudos centrados nessa temática.

A construção de vínculos entre os profissionais de enfermagem e os familiares pode amenizar as percepções negativas do ambiente hospitalar, as experiências dolorosas, bem como auxiliar os familiares no enfrentamento do medo e da angústia, inerentes ao processo de hospitalização, especialmente em UTI. Dessa forma, a comunicação pode ser um instrumento fundamental na interrelação entre profissionais de enfermagem, pacientes e familiares, possibilitando-os uma vivência mais tranqüila para todos os envolvidos.

Nessa direção, ratifica-se a necessidade de que os profissionais de enfermagem reflitam acerca de sua atuação, a fim de ampliá-la e qualificá-la, reconhecendo que junto com o paciente sob seus cuidados, há uma família que também está sofrendo e necessita de atenção e cuidados. Assim, o alcance da qualidade da assistência desejada ao paciente e seus familiares na UTI requer o envolvimento e a responsabilização dos trabalhadores.

Para isso, é necessário que os profissionais de enfermagem/saúde se identifiquem como agentes transformadores, capazes de proporcionar apoio, não somente relacionado à saúde e doença do indivíduo, mas também capazes de implementar um cuidado integral.

\section{REFERÊNCIAS}

1. Silveira RS, Lunardi VL, Filho WDL, Oliveira AMN. Uma tentativa de humanizar a relação da equipe de enfermagem com a família de pacientes internados na UTI. Texto Contexto Enferm. [periódico na Internet]. 2005;14(0) [acesso em 2008 Dez 10]. Disponível: http:// www.scielo.br/

2. Inaba LC, Silva MJP, Telles SCR. Paciente crítico e comunicação: visão de familiares sobre sua adequação pela equipe de enfermagem. Rev Esc Enferm USP. [periódico na Internet] 2005;39(4). [acesso em 2008 Dez 8]. Disponível: http://www.scielo.br/

3. Henckemaier L. Dificuldades ao cuidar da família no hospital. In: Elsen I, Marcon SS, Santos MR dos, editores. O viver em família e sua interface com a saúde e a doença. Maringá: Eduem; 2002. p.404-19.

4. Franco MC, Jorge MSG. Sofrimento do familiar frente à hospitalização. In: Elsen I, Marcon SS, Santos MR, editores. O viver em família e sua interface com a saúde e a doença. Maringá: Eduem; 2002. p.181-198.

5. Siqueira AB, Filipini R, Posso MBS, Fiorano AMM, Gonçalves SA. Relacionamento enfermeiro, paciente e família: fatores comportamentais associados à qualidade da assistência. Rev ABC [periódico na Internet]. 2006;31(2):73-7 [acesso em 2007 Mai 10]. Disponível: http://www.fmabc.br/admin/files/revistas/ 31amabc73.pdf.

6. Marcon SS, Soares NTI, Sassá AH. Users perception on their relationship with health professionals: an exploratory study. Online Braz J Nurs. [periódico na Internet]. 2007;6(0). [acesso em 2007 Ago 10]. Disponível: http://www.uff.br/objnursing/index.php/nursing/article/ view/654/154

7. Boehs AE. O sistema profissional de cuidado e a família: os movimentos de aproximação e distanciamento. In: Elsen I, Marcon SS, Santos MR, editores. O viver em família e sua interface com a saúde e a doença. Maringá: Eduem; 2002. p.247-68.

8. Pinho LB, Santos SMA. O relacionamento interpessoal como instrumento de cuidado no hospital geral. Cogitare Enferm [periódico na Internet]. 2007;12(3):377-85. [acesso em 2008 Ago 15]. Disponível: http://www.ser.ufpr.b/cogi tare

9. Lima AB, Rosa DOS. O sentido da vida familiar do paciente crítico. Rev Esc Enferm USP [periódico na Internet]. 2008;42(3). [acesso em 2008 Set 12]; Disponível: http://www.scielo.br/

10. Lima LR, Stival MM, Lima LR, Oliveira CR, Chianca 
TCM. Proposta de instrumento para coleta de dados de enfermagem em uma unidade de terapia intensiva fundamentado em Horta. Rev Eletr Enf [periódico na Internet]. 2006; 8(3):349-57. [acesso em 2007 Jun 12]. Disponível: http://www.fen.ufg.br/revista8_3/von3a05.htm.

11. Assunção AN, Paz I, Hermes CA. A visão dos enfermeiros de um hospital geral acerca da comunicação na relação interpessoal terapêutica. RECENF. 2004; 2(7): 27-32. 